University of Wollongong

Research Online

Faculty of Engineering - Papers (Archive)

Faculty of Engineering and Information

Sciences

2009

\title{
Spin wave scattering and interface magnetism in superconducting- ferromagnet-superconducting hybrid structures
}

\author{
Gustavo A. Alvarez \\ University of Wollongong, gustavo@uow.edu.au \\ Xiaolin Wang \\ University of Wollongong, xiaolin@uow.edu.au \\ T Puzzer \\ University of New South Wales \\ Germanas Peleckis \\ University of Wollongong, peleckis@uow.edu.au
}

S. X. Dou

University of Wollongong, shi@uow.edu.au

Follow this and additional works at: https://ro.uow.edu.au/engpapers

Part of the Engineering Commons

https://ro.uow.edu.au/engpapers/3238

\section{Recommended Citation}

Alvarez, Gustavo A.; Wang, Xiaolin; Puzzer, T; Peleckis, Germanas; and Dou, S. X.: Spin wave scattering and interface magnetism in superconducting-ferromagnet-superconducting hybrid structures 2009 , 07E326-1-07E326-3.

https://ro.uow.edu.au/engpapers/3238

Research Online is the open access institutional repository for the University of Wollongong. For further information contact the UOW Library: research-pubs@uow.edu.au 


\title{
AIP $\mid$ Applied Physics
}

\section{Spin wave scattering and interface magnetism in superconducting- ferromagnet-superconducting hybrid structures}

\author{
G. A. Alvarez, X. L. Wang, T. Puzzer, G. Peleckis, and S. X. Dou \\ Citation: J. Appl. Phys. 105, 07 E326 (2009); doi: 10.1063/1.3076544 \\ View online: http://dx.doi.org/10.1063/1.3076544 \\ View Table of Contents: http://jap.aip.org/resource/1/JAPIAU/v105/i7 \\ Published by the American Institute of Physics.
}

\section{Related Articles}

Ultrathin magnetic oxide EuO films on $\mathrm{Si}(001)$ using SiOx passivation-Controlled by hard $\mathrm{x}$-ray photoemission spectroscopy

J. Appl. Phys. 113, 17C505 (2013)

Heterojunction of multiferroic $\mathrm{HoMnO} 3$ on $\mathrm{Nb}$-doped $\mathrm{SrTiO} 3$

J. Appl. Phys. 113, 17C709 (2013)

The manipulation of magnetic properties by resistive switching effect in $\mathrm{CeO} 2 / \mathrm{La} 0.7(\mathrm{SrO} .1 \mathrm{Ca0} .9) 0.3 \mathrm{MnO} 3$ system

J. Appl. Phys. 113, 17C708 (2013)

Fabrication of ferrimagnetic FeOx thin film and the resistance switching of $\mathrm{Au} / \mathrm{FeOx} / \mathrm{Pt}$ heterostructure J. Appl. Phys. 113, 17C303 (2013)

Observation of the intrinsic Gilbert damping constant in $\mathrm{Co} / \mathrm{Ni}$ multilayers independent of the stack number with perpendicular anisotropy

Appl. Phys. Lett. 102, 102401 (2013)

\section{Additional information on J. Appl. Phys.}

Journal Homepage: http://jap.aip.org/

Journal Information: http://jap.aip.org/about/about_the_journal

Top downloads: http://jap.aip.org/features/most_downloaded

Information for Authors: http://jap.aip.org/authors

\section{ADVERTISEMENT}

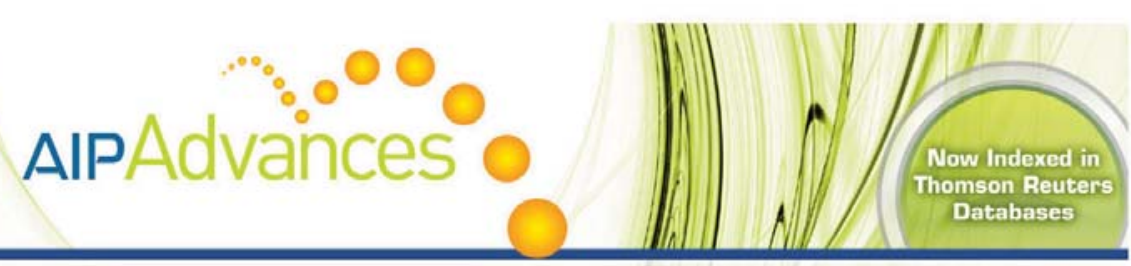
Explore AIP's open access journal:
Rapid publication
Article-level metrics
Post-publication rating and commenting 


\title{
Spin wave scattering and interface magnetism in superconducting-ferromagnet-superconducting hybrid structures
}

\author{
G. A. Alvarez, ${ }^{1,2, a)}$ X. L. Wang, ${ }^{1}$ T. Puzzer, ${ }^{3}$ G. Peleckis, ${ }^{1}$ and S. X. Dou ${ }^{1}$ \\ ${ }^{1}$ Institute for Superconducting and Electronic Materials, University of Wollongong, Innovation Campus, \\ Squires Way, Fairy Meadow, New South Wales 2519, Australia \\ ${ }^{2}$ School of Engineering Physics, University of Wollongong, New South Wales 2522, Australia \\ ${ }^{3}$ Centre of Excellence in Advanced Silicon Photovoltaics and Photonics, University of New South Wales, \\ Sydney, New South Wales 2052, Australia
}

(Presented 11 November 2008; received 2 October 2008; accepted 29 December 2008; published online 10 April 2009)

\begin{abstract}
In this work we report on interlayer spin-polarized transport studies carried out on $\mathrm{YBa}_{2} \mathrm{Cu}_{3} \mathrm{O}_{7-\delta} / \mathrm{La}_{2 / 3} \mathrm{Sr}_{1 / 3} \mathrm{MnO}_{3} / \mathrm{YBa}_{2} \mathrm{Cu}_{3} \mathrm{O}_{7-\delta}$ thin film multilayers. Anisotropic $\mathrm{YBa}_{2} \mathrm{Cu}_{3} \mathrm{O}_{7-\delta}$ high-temperature superconductors (YBCO) and the manganite $\mathrm{La}_{2 / 3} \mathrm{Sr}_{1 / 3} \mathrm{MnO}_{3}$ (LSMO) are layered metallic systems where the $c$-axis interlayer current transport occurs via sequential tunneling of charge carriers. The temperature dependence of the resistance $R(T)$ for different applied fields shows that these heterostructures are clearly influenced by both the insulator-to-metal transition of the LSMO layers at $\sim 320 \mathrm{~K}$ and the stripe fluctuations in the conductivity of the YBCO layers at the onset of electronic inhomogeneity at $\sim 210 \mathrm{~K}$. Furthermore, the tunneling of spin-polarized charge carriers between the YBCO layers through LSMO showed sharp switching between the high and low resistance states, which we assume corresponds to antiparallel and parallel alignments of the magnetic (spin) moments of these hybrid structures. The investigation of these promising hybrid structures is aimed at understanding the science of the new spin-based devices, laying the foundation for a new generation of ultrafast, nonvolatile electronics. (C) 2009 American Institute of Physics. [DOI: 10.1063/1.3076544]
\end{abstract}

\section{INTRODUCTION}

The coexistence of superconductivity and ferromagnetic (FM) phenomena under certain circumstances gives rise to novel kinds of combined ground states. ${ }^{1}$ The study and fabrication of superconducting/FM heterostructures and superlattices have become important tools in the search for novel materials for the fabrication of spintronic devices. ${ }^{2}$ When two superconductors are coupled through a conducting FM layer, the electrons sense the magnetization and are pulled apart in energy, depending on their spin orientation. As typically the superconducting critical temperature, $T_{c} \ll$ the FM Curie temperature, $T_{C}$, the coherence length of the Cooper pairs in the FM layer is short, of the order of a few nanometers. ${ }^{3}$ The reason is that a singlet Cooper pair is symmetric in the spin directions. In contrast, a ferromagnet tends to have all the spins pointing in the same direction. The exchange field within a FM layer results in decoherence of the Cooper pairs, resulting in the formation of the so-called Larkin-Ovchinnikov-Fulde-Ferrel state, ${ }^{4,5}$ in which the superconducting order parameter oscillates in sign with a period of $2 \pi \xi$ as it decays into the FM layer with a coherence length $\xi$.

Several FM oxides exhibit bulk colossal magnetoresistance (CMR), e.g., $\mathrm{La}_{2 / 3}(\mathrm{Ca}, \mathrm{Sr})_{1 / 3} \mathrm{MnO}_{3}$. These oxides, when grown as layered structures, may even show an intrinsic spin-valve effect, e.g., bilayer manganites. ${ }^{6}$ The similar

a)Author to whom correspondence should be addressed. Electronic mail: lattice constants and growth conditions of the high $T_{c}$ superconductor $\mathrm{YBa}_{2} \mathrm{Cu}_{3} \mathrm{O}_{7-\delta}(\mathrm{YBCO})$, with $T_{c}=92 \mathrm{~K}$, and the half-metal manganite compound $\mathrm{La}_{2 / 3} \mathrm{Ca}_{1 / 3} \mathrm{MnO}_{3}$ (LCMO), which exhibits CMR and a FM ground state below $T_{C}$ $=275 \mathrm{~K}$, have enabled several groups to epitaxially grow superlattices and heterostructures using various techniques, such as molecular beam epitaxy, laser ablation, or magnetron sputtering. ${ }^{7-9}$ Sefrioui et al. ${ }^{10}$ carefully studied the interplay between magnetism and superconductivity from transport and magnetization measurements in high-quality $\mathrm{YBCO} /$ LCMO superlattices; their YBCO/LCMO/YBCO heterostructures showed the existence of strong interaction between the SC and FM order parameters. Experiments with such multilayers give important information about the spindependent properties of high $T_{c}$ superconductors (HTS).

We report experimental results of interlayer magnetotransport studies carried out on YBCO and half-metal colossal magnetoresistive LSMO epitaxial thin film heterostructures. The close lattice match among the constituent layers of the perovskite YBCO/LSMO/YBCO and the substrate facilitates epitaxial film growth, thus minimizing strong spin-flip scattering at the interface and preserving spin polarization during current movement through the magnetotransport measurements.

\section{EXPERIMENTAL AND DISCUSSION}

A diagram of the YBCO/LSMO/YBCO trilayer structures used in this study is shown in the inset of Fig. 1. The samples were fabricated by pulsed laser deposition. Single 


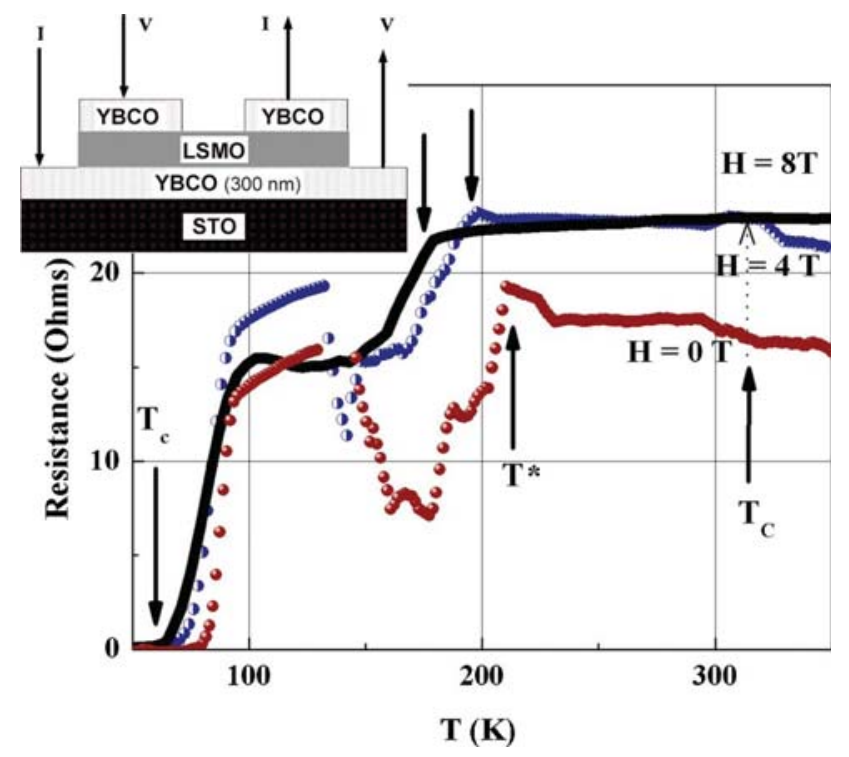

FIG. 1. (Color online) Temperature dependence of the resistance in applied fields up to $8 \mathrm{~T}, H \| c$ (YBCO/LSMO/YBCO structures). Note the onset temperature of the electronic inhomogeneity, $T^{*} \approx 210 \mathrm{~K}$. The abrupt drop in $R(t)$ at $T^{*}$ is a consistent signature of the pseudogap that is present above the superconducting transition temperature $T_{c}$. This abrupt drop is strongly reduced at fields of 4 and $8 \mathrm{~T}$, as shown by the arrows. A sketch of the sample geometry chosen for the tunneling of the spin-polarized quasiparticles along the $c$-axis is shown in the inset. The film thicknesses were 300 and $50 \mathrm{~nm}$ for the YBCO layers and the LSMO, respectively.

thin film deposition is described elsewhere. ${ }^{11}$ The magnetoresistance (MR) was measured using a standard four-probe method between 5 and $350 \mathrm{~K}$. The heterostructures were placed in the center of a superconducting solenoid with a maximum field of $8 \mathrm{~T}$. The MR was determined by applying magnetic fields perpendicular to the plane of the substrate.

It is well known that for YBCO, anti-ferromagnetic (AFM) correlations and fluctuations persist, even for optimally doped samples above $T_{c}$. ${ }^{12}$ There exists clear evidence that the charge dynamics is strongly affected by the magnetic correlations, with the most prominent example being the socalled pseudogap phenomenon. ${ }^{13,14}$ This implies that if superconductivity is driven by correlations and/or precursors of Cooper pairs around a certain temperature $T^{*}, T_{c}<T<T^{*}$, 15 the spins become paired into fluctuating singlets (quantum spin liquid). As $T$ is decreased through $T^{*}, R(T)$ drops as the spin scattering is frozen out, and the phonon contribution changes slightly, permitting the formation of this quantum spin liquid.

The temperature dependence of the resistance $R(T)$ for different applied magnetic fields $(H \| c)$ up to $8 \mathrm{~T}$ is given in Fig. 1. These hybrid heterostructures are clearly influenced by both the insulator-to-metal transition of the LSMO layer at $T_{C} \approx 320 \mathrm{~K}$ and the stripe fluctuations in the conductivity of the YBCO layers at the onset of electronic inhomogeneity below $T^{*} \approx 210 \mathrm{~K}$. The abrupt drop in $R(T)$ at $T^{*}$ is a signature of the pseudogap that is present above the superconducting transition temperature, $T_{c}$ and is strongly reduced by the applied magnetic field $H$. $H$ tends to extinguish the lowenergy partial excitation gap feature above $T_{c}$ and is related to the transition temperature $T^{*}$ via a simple Zeeman energy

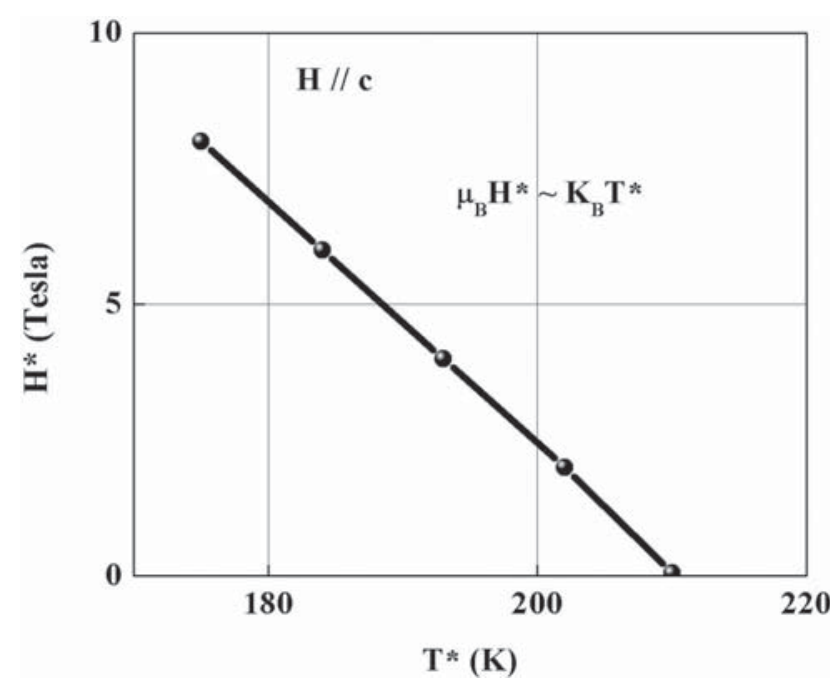

FIG. 2. The pseudogap closing field $H^{*}$ and $T^{*}$ follow a simple Zeeman scale, $\mu_{B} H^{*} \approx k_{B} T^{*}$. Here $\mu_{B}$ is the Bohr magnetron constant and $k_{B}$ is the Boltzmann constant.

scale $^{16} \mu_{B} H^{*} \approx k_{B} T^{*}$. Here $\mu_{B}$ is the Bohr magneton and $k_{B}$ is the Boltzmann constant. This is shown in Fig. 2 and is suggestive of a pseudogap dominated by the spin rather than the charge-correlation channel $^{17}$ and will be discussed elsewhere. $^{18}$

The interplay between a strong electron-phonon coupling and the double exchange effect of spin alignment on electron kinetic energy ${ }^{19}$ localizes the conduction electrons as polarons as $T$ is lowered further toward $T_{c}$. A gap opens in the electron spectrum, and $R$ rises again at 160, 140, and 120 $\mathrm{K}$ for 0,4 , and $8 \mathrm{~T}$, respectively, as shown in Fig. 1, but the polaron effect is turned off as $T$ approaches the $T_{c}$ onset. The resistance drops sharply as the gap closes, the fluctuations tend to disappear, and the spin pairs become static as they move into the superconducting state.

Figure 3 shows the MR of an YBCO/LSMO/YBCO heterostructure in magnetic fields up to $5 \mathrm{~T}$ at $140 \mathrm{~K}$. The bias currents were 5, 550, and $1050 \mu \mathrm{A}$. Below $0.7 \mathrm{~T}$ the resis-

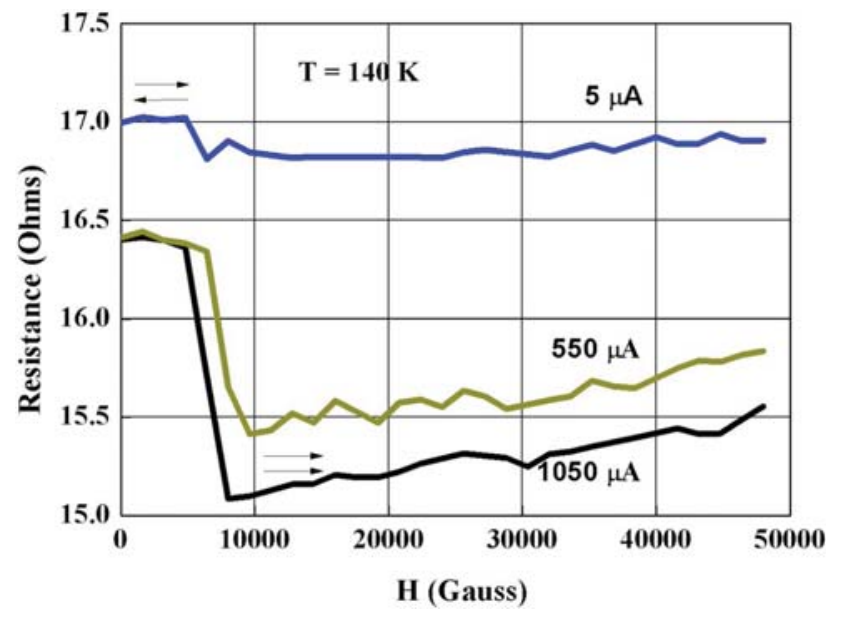

FIG. 3. (Color online) MR effect in YBCO/LSMO/YBCO structures in fields up to $5 \mathrm{~T}\left(H \|_{c}\right)$ at $140 \mathrm{~K}(1 \mathrm{~T}=10000 \mathrm{G})$. Note that the magnetization vectors switch from antiparallel to parallel at some critical field around $0.7 \mathrm{~T}$. 


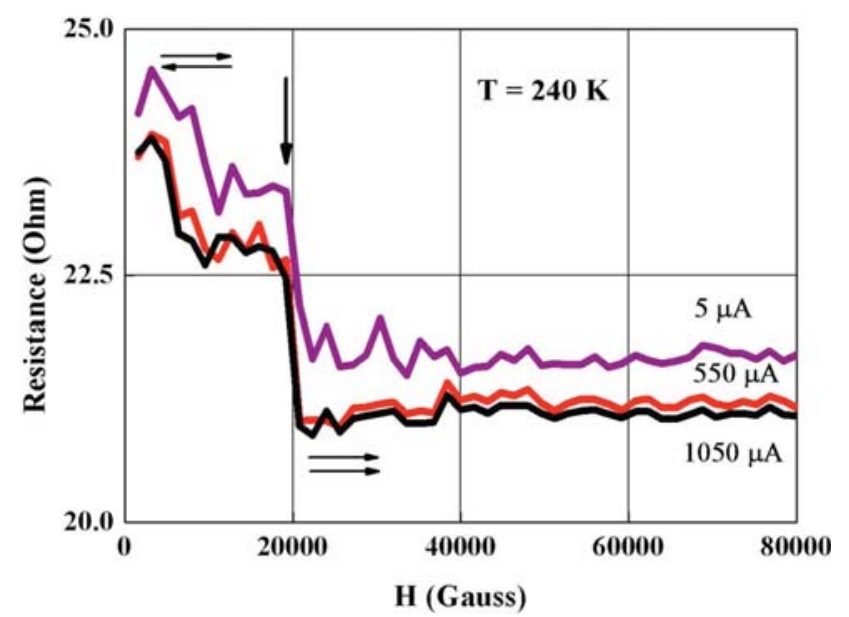

FIG. 4. (Color online) MR effect in YBCO/LSMO/YBCO structures in fields up to $8 \mathrm{~T}(H \| c)$ at $240 \mathrm{~K}(1 \mathrm{~T}=10000 \mathrm{G})$. The magnetization vectors switch fully from antiparallel to parallel at some critical field around 2 $\mathrm{T}$ (marked by the arrow), and the MR becomes constant.

tance is almost constant for the different bias currents. The magnetization vectors switch from antiparallel to parallel at some critical field around $0.7 \mathrm{~T}$, leading to a discontinuous jump in the MR. The resistance dropped to about $1 \%, 6 \%$, and $9 \%$ of its zero-field value for 5, 550, and $1050 \mu \mathrm{A}$ bias currents, respectively. Such behavior demonstrates the strong effect that Lorentz forces exert in the MR tunneling process. The MR becomes larger when the applied magnetic field $(H \| c)$ is decreased, and it increases almost linearly with the applied current.

Figure 4 shows the MR of an YBCO/LSMO/YBCO heterostructure at $240 \mathrm{~K}$. In small fields $(H<1 \mathrm{~T})$, the resistance drops continuously with increasing applied field, as, it appears, the magnetization vectors are smoothly tilted away from their antiparallel alignment along the $c$-axis toward a parallel orientation, leading to a continuous evolution of the MR, and the MR becomes constant. The resistance drops to about $12 \%$ of its zero-field value for different bias currents. Opposite to the case at $140 \mathrm{~K}$, the MR process is not influenced by Lorentz forces at temperatures $T>T^{*}$.

The LSMO has a finite density of states near the Fermi level for both spin polarizations, although the spin mobility is much higher for the majority spins, ${ }^{20}$ so interplane transport of the spin-polarized charge carriers occurs via tunneling processes, leading to a high-temperature tunneling MR. Therefore, it is highly possible that spin diffusion (driven by the gradient in spin polarization between LSMO and YBCO and opposed to the relaxation in the YBCO layer) may lead to a long-range spin polarization of the charge carriers deep inside the YBCO layers at temperatures above $T_{c}$.

In summary, the magnetic field dependence of $R(T)$ demonstrates that the magnetic field $H$ is related to the onset temperature $\left(T^{*}\right)$ of the electronic inhomogeneity via a simple Zeeman energy scale, suggestive of a pseudogap dominated by the spin rather than the charge-correlation channel. The interplay of electron-phonon coupling and double exchange can account for the dramatic changes in the resistance close to $T_{c}$ and the extreme sensitivity to magnetic field.

The transport of spin-polarized charge carriers between the YBCO layers coupled by the half-metallic FM LSMO leads to switching effects of the MR at temperatures above $T_{c}$. This spin-flip switching from antiparallel to parallel states was indicated by the sharp jumps in the MR and occurs for a field where the Zeeman energy overcomes the interlayer coupling energy. ${ }^{21}$

The large jump in the resistance observed for different samples means that hopping of electrons along the $c$-axis of the LSMO layer is affected by the relative alignment of the core spins, being maximal when the core spins are parallel and minimal when they are antiparallel; The role of spin in these hybrid heterostructures is extremely important for potential applications ranging from spin-based electronics to quantum computers, or for the design of devices based on magnetic switching processes.

\section{ACKNOWLEDGMENTS}

This work was partially supported by the Australian Research Council. We are grateful to Dr. T. Silver and Dr. J. Horvat for useful discussions. We also thank Professor C. Cook for his support and advice.

${ }^{1}$ L. N. Bulaevskii, A. I. Buzdin, M. L. Kulic, and S. V. Panjukov, Adv. Phys. 34, 175 (1985).

${ }^{2}$ M. Kontos, Phys. Rev. Lett. 86, 304 (2001).

${ }^{3}$ S. A. Wolf, D. D. Awschalom, R. A. Buhrman, J. M. Daughton, S. von Molnar, M. L. Roukes, A. Y. Chtchelkanova, and D. M. Treger, Science 294, 1488 (2001).

${ }^{4}$ A. I. Larkin and Y. N. Ovchinnikov, Sov. Phys. JETP 20, 762 (1964).

${ }^{5}$ P. Fulde and R. A. Ferrel, Phys. Rev. 135, A550 (1964).

${ }^{6}$ T. Nachtrab, S. Heim, M. Mößle, R. Kleiner, O. Waldmann, R. Koch, P. Müller, T. Kimura, and Y. Tokura, Phys. Rev. B 65, 012410 (2001).

${ }^{7}$ I. Bozovic, IEEE Trans. Appl. Supercond. 11, 2686 (2001).

${ }^{8}$ P. Prieto, P. Vivas, G. Campillo, E. Baca, L. F. Castro, M. Varela, C. Ballesteros, J. E. Villegas, D. Arias, C. León, and J. Santamaría, J. Appl. Phys. 89, 8026 (2001).

${ }^{9}$ H.-U. Habermeier, G. Cristiani, R. K. Kremer, O. Lebedev, and G. Van Tendeloo, Physica C 364-365, 298 (2001); S. Soltan, J. Albrecht, and H.-U. Habermeier, Phys. Rev. B 70, 144517 (2004).

${ }^{10}$ Z. Sefrioui, D. Arias, V. Peña, J. E. Villegas, M. Varela, P. Prieto, C. León, J. L. Martinez, and J. Santamaria, Phys. Rev. B 67, 214511 (2003).

${ }^{11}$ G. A. Alvarez and M. Koyanagi, Cryogenics 35, 361 (1995); Y. Konishi, T. Kimura, M. Izumi, M. Kawazaki, and Y. Tokura, Appl. Phys. Lett. 73, 3004 (1998).

${ }^{12}$ J. Orenstein and A. J. Millis, Science 288, 468 (2000).

${ }^{13}$ E. V. L. de Mello, E. S. Caixero, and J. L. Gonzalez, Phys. Rev. B 67, 024502 (2003).

${ }^{14}$ C. Timm, D. Manske, and K. H. Bennemann, Phys. Rev. B 66, 094515 (2002).

${ }^{15}$ Q. Si, Phys. Rev. Lett. 78, 1767 (1997).

${ }^{16}$ T. Shibauchi, L. Krusin-Elbaum, and M. P. Ming Li, Phys. Rev. Lett. 86, 5763 (2001).

${ }^{17}$ Y.-J. Kao, A. P. Iyengar, Q. Chen, and K. Levin, Phys. Rev. B 64, 140505 (2001).

${ }^{18}$ G. A. Alvarez and T. Puzzer (unpublished).

${ }^{19}$ P. Pieri, G. C. Strinati, and D. Moroni, Phys. Rev. Lett. 89, 127003 (2002).

${ }^{20}$ B. Nadgorny, I. I. Mazin, M. Osofsky, R. J. Soulen, P. Broussard, R. M. Stroud, D. J. Singh, V. G. Harris, A. Arsenov, and Ya. Mukovskii, Phys. Rev. B 63, 184433 (2001).

${ }^{21}$ A. J. Millis, R. Mueller, and B. I. Shraiman, Phys. Rev. B 54, 5405 (1996). 